\title{
Implementación de un servicio de monitoreo y control de jardines en un municipio de Lima mediante el uso de Internet de las Cosas
}

\author{
Jorge Guerra Guerra \\ jguerrag@unmsm.edu.pe / Universidad Nacional Mayor de San Marcos. Lima, Perú \\ Yessenia Lunarejo Gonzales \\ yessenia.lunarejo@unmsm.edu.pe / Universidad Nacional Mayor de San Marcos. Lima, Perú \\ Armando Fermín Pérez \\ fferminp@unmsm.edu.pe / Universidad Nacional Mayor de San Marcos. Lima, Perú
}

Recepción: 1-7-2018 / Aceptación: 20-8-2018

Resumen. El presente trabajo muestra la implementación de un sistema de monitoreo basado en tecnología Open Hardware, destinado a la gestión de jardines de un municipio de la capital peruana, usando red móvil y sensores para el manejo apropiado del agua con datos en tiempo real.

PALABRAS ClaVE: monitoreo ambiental, Internet de las Cosas, gestión de jardines

Implementation of a monitoring and control service for the gardens of a municipality in Lima using the Internet of Things

ABstract. The following goes over the implementation of a monitoring system using Open Hardware. The latter will be set up at a public garden in Lima, using sensors and the local mobile network, for municipal water management and distribution (metering) in real time.

KEYWORDS: environmental monitoring, Internet of Things, garden management 


\section{INTRODUCCIÓN}

El problema del uso de agua en parques públicos es un tema recurrente en trabajos tanto de organizaciones que abordan temas de medio ambiente como en la industria en general. Así por ejemplo, en Thamaraimanalan, Vivekk, Satheeshkumar y Saravanan (2018) se detalla la implementación de un sistema automático de monitoreo de plantas y control de riego de jardines públicos para mejorar la calidad de vida de la población. Sandhya, Pallavi y Chandrashekar (2017) proponen algo similar a nivel de jardines de las casas utilizando diversas tecnologías de comunicación como wifi, redes móviles y la nube.

El problema en estudio en este artículo se registra en la municipalidad distrital de San Isidro, que tiene acceso a la distribución del agua del río Surco, donde, luego de un proceso de decantación, puede ser usada en el riego de áreas verdes. Sin embargo, esta cantidad de agua no cubre la totalidad de la demanda de riego de las áreas verdes del distrito, por lo que debe cubrirse con agua potable, resultando así en un incremento en los gastos de mantenimiento de las áreas verdes.

Son dos los métodos empleados para el riego de áreas verdes en el distrito: inundación (25 parques) y tecnificado mediante aspersores (14 parques). Los criterios para regar los parques en general se basan en el procedimiento de evapotranspiración, según lo indica el Área de Sostenibilidad de la Municipalidad. Con base en ello se estableció un horario de riego para todo el distrito: lunes de 6:00 a 18:00 horas y jueves de 18:00 a 6:00 horas. Sin embargo, se riega con procedimientos y criterios más intuitivos que formales debido a la deficiente o nula capacitación e información técnica disponible; de allí la necesidad de contar con una solución tecnológica que cubra la gestión y control de los jardines para manejar de manera eficiente el uso del agua destinada a esta tarea.

El resto del artículo está organizado de la siguiente manera, en la sección 2 se presenta una propuesta de implementación; en la sección 3 se muestran los resultados preliminares, mientras que en la sección 4 se describen las conclusiones y futuros trabajos.

\section{Metodología}

La gestión del riego de los parques públicos del caso en estudio enfrenta dos retos: uno es el uso deficiente del agua, ya que no se cuenta con datos concretos para conocer si varios de los parques regados por inundación están siendo sobrerregados o regados cuando no es necesario, y otro es el incremento en los costos de mantenimiento de las áreas verdes debido a un mayor uso de agua potable. Para este problema se han desarrollado soluciones basadas en Internet de las Cosas (IoT por sus siglas en inglés) como la propuesta por Bermúdez (2014), que plantea una solución de riego automatizada usando Arduino, o la propuesta por 
Angelopoulos, Nikoletseas y Theofanopoulos (2011) usando comunicación XBEE, lo que permite transferir los datos a un centro de información para su posterior procesamiento.

Las soluciones tecnológicas basadas en redes inalámbricas cuentan con herramientas apropiadas como la indicada por Khan, Islam y Hafeez (2011), que muestran un modelo para pronóstico de la demanda de agua para riego mejorado por los mismos autores (2012), donde muestran alternativas basadas en modelos estadísticos y de inteligencia artificial; debe indicarse, además, el trabajo de Karray et al. (2016), que define una red de sensores para el monitoreo de agua que circula por las tuberías. Las condiciones planteadas para la gestión de jardines de la municipalidad de San Isidro son las siguientes, estableciendo el control del estado del suelo:

i. Capacidad de campo: definiendo los distintos tipos de suelo en los parques, poner sensores que puedan medir las variables que impactan la capacidad del suelo para cada parque, y así evaluar cuál es la necesidad de riego de cada parque. La cantidad de sensores dependería de la cantidad de zonas y parques con distintos suelos.

ii. Medición de evapotranspiración (ETo): esta permitiría saber cuánto varía la humedad del suelo para así determinar qué parques han perdido más humedad o cuáles necesitan mayor recuperación de humedad. Esto se puede hacer por zonas.

iii. Medición del clima: tener estaciones climáticas tanto (de preferencia) en la parte alta como en la parte baja para medir viento (velocidad y dirección), horas de sol, humedad, temperatura, Eto; es decir, condiciones climáticas para los cultivos (se realizará en fase II).

iv. Se plantea como solución obtener los valores de las siguientes mediciones, al inicio:

- ETo.

- Variación de la humedad del suelo.

- Humedad relativa.

- Temperatura.

Considerando la tecnología a utilizar (preferentemente Open Hardware) y usando tecnología inalámbrica y procesamiento en nube, y Edge Computing; el modelo planteado sería como muestra la figura 1 . 


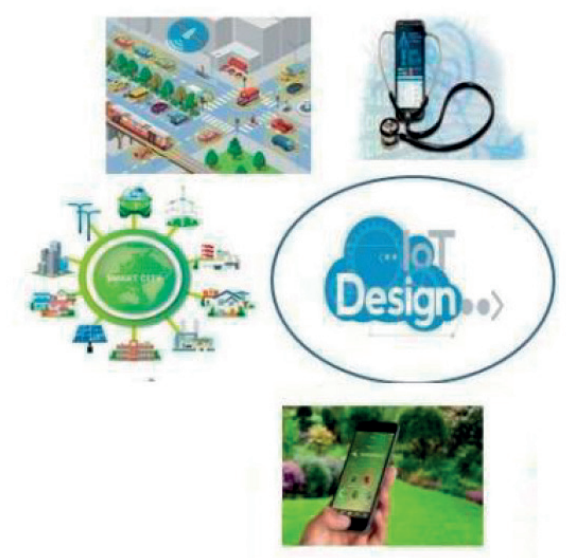

Figura 1. Modelo general de gestión de riego usando IoT Elaboración propia

Para esta implementación se está desarrollando la configuración de nodos de sensores para los jardines asignados que muestra la figura 2.

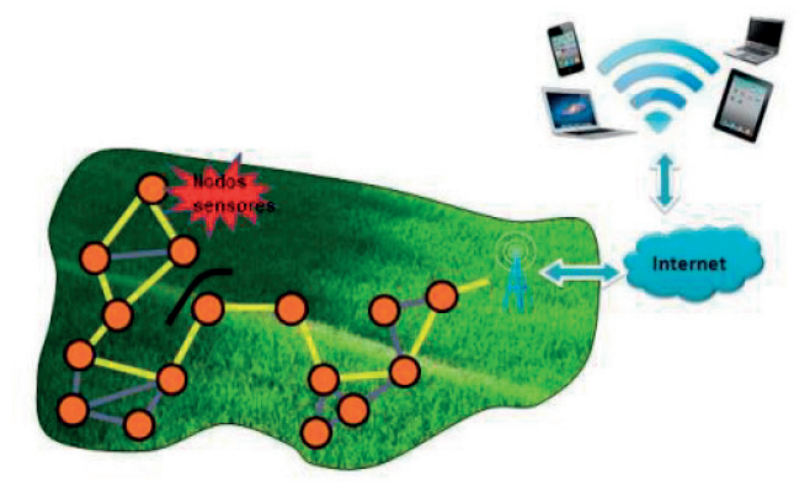

Figura 2. Distribución de sensores en jardines de San Isidro usando red inalámbrica Elaboración propia

El número de sensores por jardín depende de la extensión que tengan. En los trabajos previos se ha determinado de que cada nodo cubrirá un área aproximada de $250 \mathrm{~m}^{2}$, ajustándose de acuerdo al tipo de terreno y vegetación. La implementación de dichos nodos, considerados en esta propuesta, será realizada de acuerdo al modelo de la figura 3. 


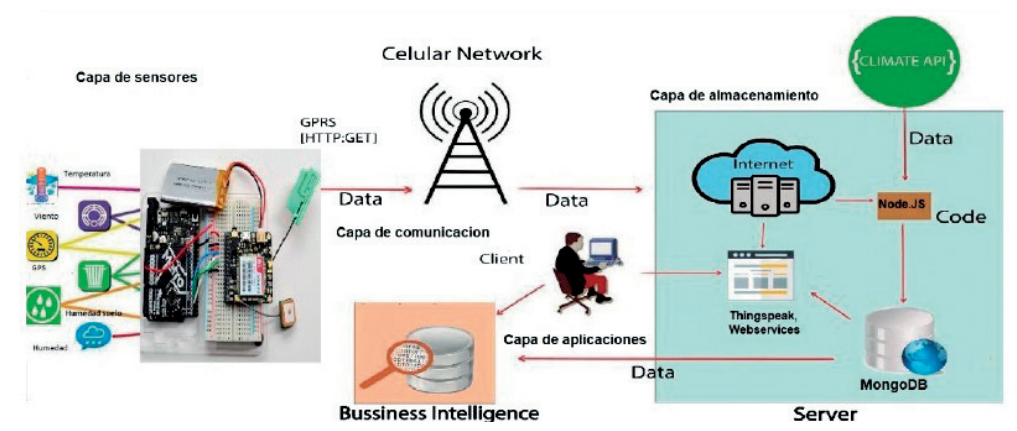

Figura 3. Modelo de nodo conectado a red inalámbrica

Elaboración propia

En la implementación de la solución comparamos dos modelos posibles de desarrollo.

\subsection{Implementación basada en comunicación wifi}

Esta implementación considera el uso de wifi donde esté disponible, usando para ello el módulo ThingDev, basado en esp8266. El diagrama de implementación obtenido se muestra en la figura 4.

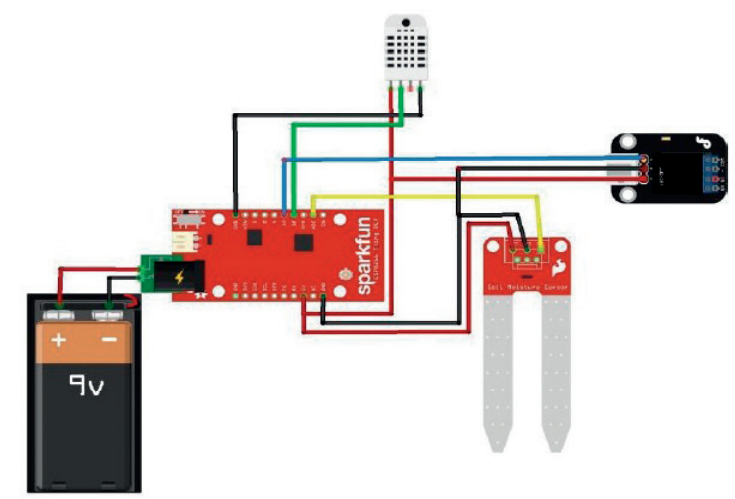

Figura 4. Propuesta de solución usando conexión wifi Elaboración propia

La nube que registra los datos obtenidos por el modelo implementado es Thingspeak (https://thingspeak.com/login), para la cual se implementaron dos canales: datos obtenidos por el modelo wifi (https://thingspeak.com/channels/529660) y datos obtenidos por el modelo GSM (https://thingspeak.com/channels/529617). 
Durante las pruebas de conexión usando la nube de Thingspeak se obtuvieron los valores de medición para la propuesta basada en wifi de la figura 5.

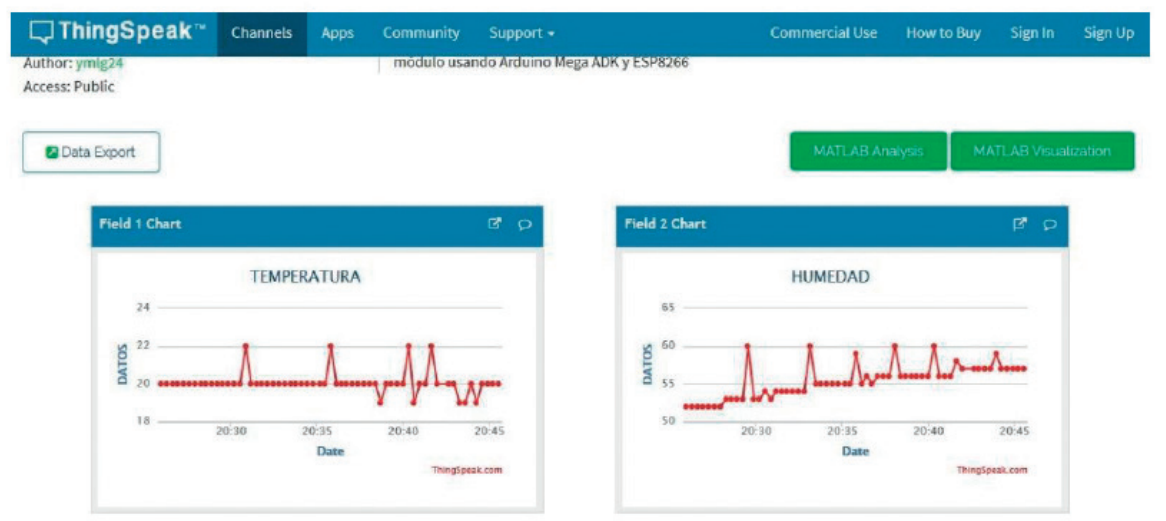

Figura 5. Canal implementado en Thingspeak, mostrando los datos obtenidos por ThingDev Elaboración propia

\subsection{Implementación basada en comunicación GSM}

En esta propuesta la comunicación de los nodos definidos con la nube donde se almacenarán los datos será mediante la red móvil disponible en la ciudad de Lima. En esta implementación el desarrollo se realizó mediante el uso del módulo Adafruit Feather 32u4, que trabaja como mínimo en $2 \mathrm{G}$ y, basado en ello, el modelo propuesto está definido en la figura 6 .

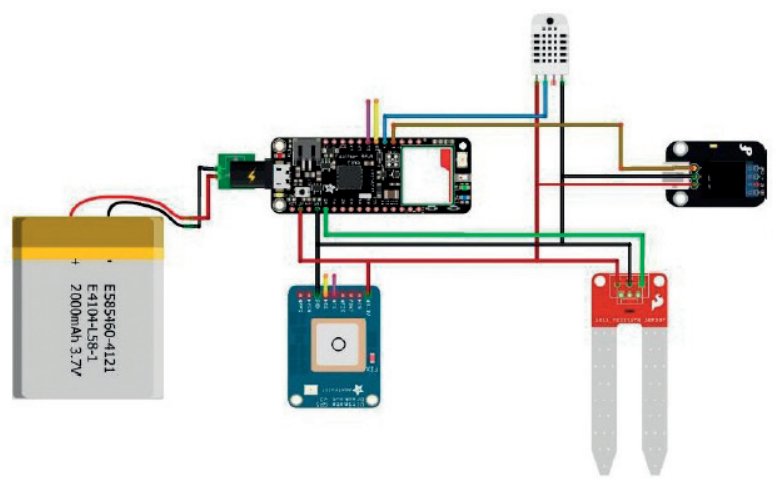

Figura 6. Propuesta de solución usando conexión con red GSM Elaboración propia 
Para esta implementación, teniendo como base la nube de sensores Thingspeak, obtenemos las mediciones que se muestran en la figura 7.

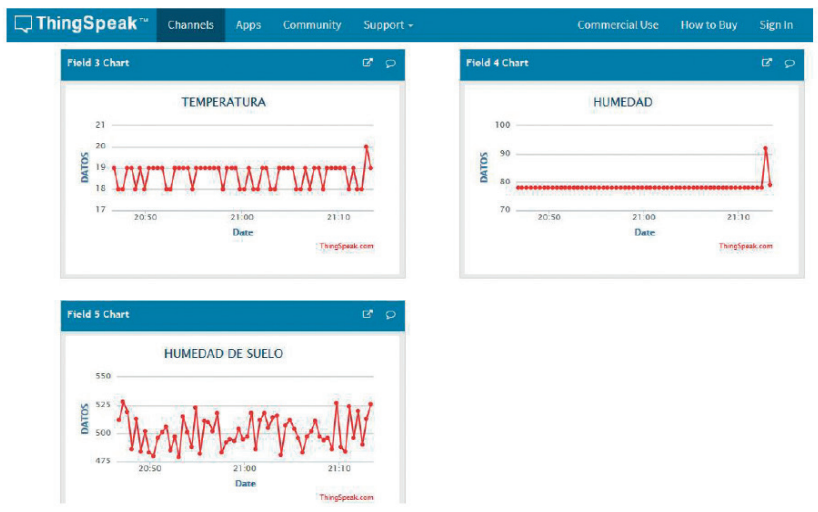

Figura 7. Mediciones de datos para el modelo usando GSM Elaboración propia

\section{Resultados}

Una vez implementados los dos modelos, se recolectan los datos encontrados y con ellos se define una tabla, en este caso con ThingDev (tabla 1).

Tabla 1

Datos obtenidos por el modelo basado en ThingDev (Thingspeak, fragmento)

\begin{tabular}{|c|c|c|c|c|c|}
\hline Fecha & Hora (UTC) & ID & $\begin{array}{c}\text { Temperatura } \\
\text { Grados } \\
\text { centígrados }\end{array}$ & $\begin{array}{c}\text { Humedad } \\
\%\end{array}$ & $\begin{array}{c}\text { Humedad } \\
\text { de suelo } \\
\text { L/m² }\end{array}$ \\
\hline $1 / 7 / 2018$ & $0: 59: 25$ & 1 & 21 & 38 & 438 \\
\hline $1 / 7 / 2018$ & $0: 59: 45$ & 2 & 21 & 38 & 471 \\
\hline $1 / 7 / 2018$ & $1: 00: 05$ & 3 & 23 & 44 & 508 \\
\hline $1 / 7 / 2018$ & $1: 00: 26$ & 4 & 21 & 39 & 524 \\
\hline $1 / 7 / 2018$ & $1: 00: 47$ & 5 & 21 & 39 & 497 \\
\hline $1 / 7 / 2018$ & $1: 01: 10$ & 6 & 21 & 39 & 510 \\
\hline $1 / 7 / 2018$ & $1: 01: 28$ & 7 & 21 & 39 & 494 \\
\hline $1 / 7 / 2018$ & $1: 01: 46$ & 8 & 21 & 40 & 509 \\
\hline
\end{tabular}

(continúa) 
(continuación)

\begin{tabular}{|l|c|c|c|c|c|}
\hline $1 / 7 / 2018$ & $1: 02: 07$ & 9 & 21 & 40 & 510 \\
\hline $1 / 7 / 2018$ & $1: 02: 25$ & 10 & 21 & 40 & 527 \\
\hline $1 / 7 / 2018$ & $1: 02: 46$ & 11 & 21 & 40 & 504 \\
\hline $1 / 7 / 2018$ & $1: 03: 05$ & 12 & 21 & 41 & 518 \\
\hline $1 / 7 / 2018$ & $1: 03: 25$ & 13 & 21 & 45 & 494 \\
\hline $1 / 7 / 2018$ & $1: 03: 45$ & 14 & 21 & 41 & 501 \\
\hline $1 / 7 / 2018$ & $1: 04: 04$ & 15 & 21 & 42 & 494 \\
\hline
\end{tabular}

Elaboración propia

Tabla 2

Datos obtenidos con el módulo FONA usando red GSM (Thingspeak, fragmento)

\begin{tabular}{|c|c|c|c|c|c|}
\hline FECHA & HORA (UTC) & ID & $\begin{array}{c}\text { Temperatura } \\
\text { Grados celsius }\end{array}$ & $\begin{array}{c}\text { Humedad } \\
\%\end{array}$ & $\begin{array}{c}\text { Humedad } \\
\text { del suelo L/m² }\end{array}$ \\
\hline $1 / 07 / 2018$ & $0: 59: 10$ & 1 & 20 & 71 & 517 \\
\hline $1 / 07 / 2018$ & $0: 59: 40$ & 2 & 20 & 71 & 503 \\
\hline $1 / 07 / 2018$ & $1: 00: 21$ & 3 & 20 & 71 & 511 \\
\hline $1 / 07 / 2018$ & $1: 00: 50$ & 4 & 20 & 73 & 482 \\
\hline $1 / 07 / 2018$ & $1: 01: 16$ & 5 & 20 & 72 & 503 \\
\hline $1 / 07 / 2018$ & $1: 01: 43$ & 6 & 19 & 74 & 512 \\
\hline $1 / 07 / 2018$ & $1: 02: 10$ & 7 & 21 & 75 & 491 \\
\hline $1 / 07 / 2018$ & $1: 02: 36$ & 8 & 21 & 74 & 505 \\
\hline $1 / 07 / 2018$ & $1: 03: 03$ & 9 & 20 & 74 & 485 \\
\hline $1 / 07 / 2018$ & $1: 03: 30$ & 10 & 21 & 75 & 493 \\
\hline $1 / 07 / 2018$ & $1: 03: 57$ & 11 & 21 & 75 & 521 \\
\hline $1 / 07 / 2018$ & $1: 04: 24$ & 12 & 19 & 87 & 496 \\
\hline $1 / 07 / 2018$ & $1: 04: 50$ & 13 & 20 & 80 & 483 \\
\hline $1 / 07 / 2018$ & $1: 05: 17$ & 14 & 20 & 21 & 504 \\
\hline $1 / 07 / 2018$ & $1: 05: 44$ & 15 & & 75 & 482 \\
\hline
\end{tabular}

Elaboración propia

Con estos datos se pueden determinar dos cosas importantes: los datos (salvo en el caso de la humedad relativa) son básicamente similares, las diferencias en el caso de la humedad se deben a que un módulo está usando un sensor DHT22 mientras el otro modulo posee un 
sensor DHT11, menos preciso que el anterior. El segundo resultado que puede obtenerse es que ambos módulos pueden ser una alternativa viable y de bajo costo en una localidad donde exista wifi público, como son los distritos de San Isidro, Miraflores, Surco y otros. Sin embargo, al generalizar el modelo y tratar de aplicar este prototipo a Lima en su conjunto, la única alternativa viable es la de usar red GSM, que se encuentra extendida en todo el país, dado que una alternativa basada en fibra óptica es de un precio muy elevado y las redes LPWAN todavía están en estado de construcción y despliegue.

\section{Conclusiones}

El presente proyecto, que se encuentra en implementación, permite obtener datos en tiempo real de los nodos de gestión de jardines para su posterior envío a repositorio mediante nube pública. Se muestra además que el uso de red wifi o red móvil es indistinto. Sin embargo, para un escalamiento de la solución a nivel de toda la ciudad de Lima, la red móvil es claramente el medio apropiado para la comunicación de los sensores con el repositorio en la nube, apuntando en el futuro a ser energizado mediante paneles solares, como lo indican Difallah, Benahmed, Draoui y Bounaama (2018).

En un futuro trabajo se establecerá un repositorio de datos en la Plataforma de Interoperabilidad del Estado (PIDE), como lo está haciendo actualmente el equipo que está trabajando esta propuesta en otros proyectos. Una vez almacenada la información en base de datos NoSQL (MongoDb) se realizará el procesamiento usando técnicas de big data o analytics, según sea el caso.

\section{REFERENCIAS}

Angelopoulos, C. M., Nikoletseas, S., y Theofanopoulos, G. C. (2011). A smart system for garden watering using wireless sensor networks. En: Proceedings of the 9th ACM international symposium on Mobility management and wireless access (pp. 167-170). ACM. DOI: 10.1145/2069131.2069162

Bermúdez, D. (2014). Riego de huerta automatizado por Arduino. Tesis para optar el título de ingeniero técnico industrial eléctrico. Universidad Pública de Navarra, España.

Difallah, W., Benahmed, K., Draoui, B., y Bounaama, F. (2018). Design of a solar powered smart irrigation system (SPSIS) using WSN as an IoT device. En: Proceedings of the 2018 International Conference on Software Engineering and Information Management (pp. 124-128). ACM. DOI: 10.1145/3178461.3178482 
Karray, F., Garcia-Ortiz A., Jmal, M.W., Obeid, A.M., y Mohamed A. (2016). EARNPIPE: A Testbed for Smart Water Pipeline Monitoring Using Wireless Sensor Network. Procedia Computer Science, 96, 285-294.

Khan, M. A., Islam, M. Z., y Hafeez, M. (2012). Evaluating the performance of several data mining methods for predicting irrigation water requirement. En: Proceedings of the Tenth Australasian Data Mining Conference-Volume 134 (pp. 199-207).

Khan, M. A., Islam, Z., y Hafeez, M. (2011). Irrigation water demand forecasting: a data preprocessing and data mining approach based on spatio-temporal data. En: Proceedings of the Ninth Australasian Data Mining Conference, Volume 121 (pp. 183-194).

Sandhya, B.R., Pallavi, M., y Chandrashekar, M. (2017). IoT Based smart home garden watering system using Raspberry Pi 3. International Journal of Innovative Research in Science, Engineering and Technology, 6(12).

Thamaraimanalan, T., Vivekk, S. P., Satheeshkumar, G., y Saravanan, P. (2018). Smart Garden Monitoring System Using IOT. Asian Journal of Applied Science and Technology (AJAST), 2(2), 186-192. 